\title{
The combined finishing of geometrically-complex parts
}

\author{
Vladislav Smolentsev ${ }^{1}$, Oleg Kirillov ${ }^{1, *}$, and Vasily Kotukov ${ }^{1}$ \\ ${ }^{1}$ Voronezh State Technical University, 394026, Moskovsky Prospekt, 14, Voronezh, Russian Federation
}

\begin{abstract}
The paper describes the combined hydroabrasive dimensional processing, which involves the application of an electric field of metal parts with complex volumetric profiles, requiring jet control with reference to 5-6 coordinates. One should also consider the reciprocal impact of technological parameters upon formation of parts machined. The mechanism of dimensional material removal has been developed, which is the basis for working out the informational technology of a combined process. The objects of study are adjoining parts of spatially complex surfaces, like gas-turbine engine blades, or axial and radial wheels of turbopump units, as well as the processes of unsoldering in cooled parts of combustion chambers, jet nozzles and complex-shaped part sections with limited tool access into the processing zone, including those made from materials of low machinability. The combined process of hydroabrasive finishing has been developed, which involves the induction of electric current for geometrically-complex surfaces of any geometry, including in areas, inaccessible or hardly accessible for a metal-cutting tool when manufacturing parts from alloys of low machinability.
\end{abstract}

\section{Introduction}

The dimensional machining of geometrically-complex parts encounters great difficulties in formation of precision transitional zones. These include the rounded edges of gas-turbine engine blades with high twist angles, where the applicable string method of machining by abrasive belts makes it difficult to copy the spatially variable profile with a tool. The more precise production of such sections by narrow strips results in drastic decrease in performance, accuracy of parts and tool durability.

Apart from that, the applied methods of manufacturing edges and coupling zones of turbopump unit blades are rather labour-intensive and unstable, since these zones are hardly accessible for an instrument, requiring the unproductive manual work to ensure the desired profile precision. This hinders the fulfillment of the necessary tactical, technical and operational requirements for newgeneration transport equipment.

Jet methods, including the combined hydroabrasive machining, make it possible to switch from the string method, involving the consecutive formation of separate part zones (mainly, accessible for a tool), to pointwise remote shaping of surfaces of any profile. The presence of an electric field provides the opportunity to fully exclude impregnation and perform the finishing operations on parts of any geometry, including those with areas hidden within a product.

The modern designs of high-tech transport equipment contain a great number of high-precision elements [1, 2, 3], having no direct access by a metal-cutting tool, and the development of new technological methods and devices for their finishing considerably expands the possibilities of manufacturers of perspective machine -building products. It has required the scientific rationale and the assessment of capability for dimensional hydroabrasive machining of geometrically-complex metal parts, using the accumulated knowledge of combined processes with ensuring the technological parameters of finishing for geometrically-complex surfaces, including those having a spatial geometry.

\section{The state of studies in the area under consideration}

The analysis of available publications [3, 4] on the subject of the article has shown that the issues related to combined hydroabrasive finishing are insufficiently addressed in the domestic and translated technical literature, though the principal possibility of effective implementation of the method is shown by scientists and specialisits. Therefore, it is quite reasonable to set a goal of implementing new technological processes and devices, the emergence of which provides the possibility of finishing geometricallycomplex surfaces of metal parts. At the same time, the current scientific groundwork [5-11] is sufficient for developing new methods and devices, ensuring the attainment of the stated goal. It demands the theoretical studies on developing the mathematical apparatus for establishing the relationship of the profiles of any processing complexity with calculated and designated parameters of the combined process.

\footnotetext{
Corresponding author: kirillov.olli@yandex.ru
} 


\section{The mechanism of dimensional material removal using the combined method}

With reference to finishing, the application of an electric field helps to ensure the required accuracy of the part profile and the quality of the surface layer. It controls the boundary of the jet cross-section and the electric modes of combined machining, eliminates the impregnation (particularly, viscous and also heat-resistant materials), and reduces the surface roughness.

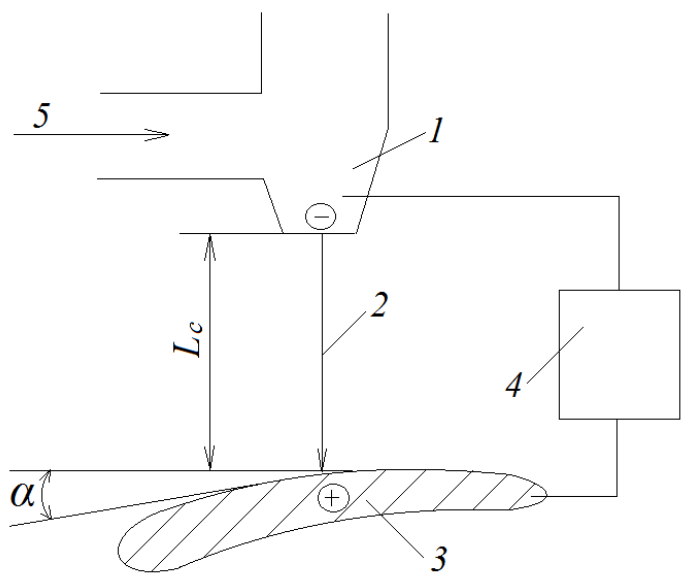

Fig. 1. The scheme of combined hydroabrasive machining: 1 - the nozzle for operating medium supply; 2 - the jet of operating medium; 3 - the machined part (anode); 4 - the source of direct current; 5 - the channel for current-conducting pellets and fluid (if necessary); $L_{c}$ - the jet length; $a$ - the angle of jet attack.

The figure 1 presents the scheme of combined hydroabrasive machining, in which the research is focused at parameters of an electric field and a jet.

The length of the undisturbed part of a jet with clear boundaries, ensuring the required accuracy of coupling between the geometrically-complex surfaces, is calculated using the formula:

$$
L_{H}=K_{H} d_{2}\left(1+\frac{1}{\sqrt{\frac{D_{c} \gamma_{c} \sigma_{c}}{\mu_{c}}}}\right) \sqrt{\frac{v_{c}^{2} D_{c} \gamma_{c}}{\sigma_{c}}},
$$

where $K_{n}$ - the numerical coefficient, considering the properties of the operating medium; $d_{\mathrm{g}}-$ the equivalent diameter of abrasive pellets, $\mathrm{mm} ; D_{c}$ - the diameter of the injector nozzle, $\mathrm{mm} ; \gamma_{c}-$ the average density of the operating medium in a jet, $\mathrm{kg} / \mathrm{m}^{3} ; \sigma_{c}$ - the surface tension coefficient of the operating medium, N/m; $\mu_{c}-$ the dynamic viscosity coefficient of the operating medium, $\mathrm{Pa} / \mathrm{s} ; v_{c}$ - the average speed of the operating medium in a jet, $\mathrm{m} / \mathrm{s}$.

The condition for dimensional machining is

$$
L c<L n
$$

The distance from the injector nozzle section to the workpiece machining zone is determined by the drawing of the part.

To regulate the dimensional material removal by a jet with hard abrasive, it is necessary to set a half-angle of jet spray, which depends on the jet pressure $\mathrm{P}_{\mathrm{c}}$

$$
P_{c}=\sqrt{\left(Q_{c} \gamma_{c} \vec{v}_{1}\right)^{2}+\left(Q_{c} \gamma_{c} \vec{v}_{2}\right)^{2}} \cdot \sin \beta \text {, }
$$

where $Q_{c}$ - the volume flow of the operating medium, $\mathrm{m}^{3} / \mathrm{s} ; \gamma_{c}-$ the density of the medium, $\mathrm{g} / \mathrm{cm}^{3} ; v_{l}-$ the jet speed before contact with the surface machined; $v_{2}-$ the flow speed after leaving the processing zone; $\beta$ - the angle of an attack, grad.

To ensure the conditions for getting finishing parameters, it is necessary to set the voltage $U$, applied to the jet, and the current I, required for anode process flow.

$$
U=\left\{\frac{j_{\kappa p} L_{c}}{1-\frac{3 \varphi_{c}}{2+\varphi_{c}-0,394 \varphi_{c}^{10 / 3}}}\right\} \chi_{0}^{-1}
$$

where $j_{k p}$ - the critical value of current density, beyond which the anodic removal of metal is possible; $L_{\mathrm{c}}-$ the distance from the nozzle section to the processing point; $\varphi_{c}$ - the level of filling the electrolyte with currentconducting particles; $\chi_{0} \quad-$ the specific electric conductivity of the operating medium before the start of machining (as measured).

The current I, supplied to the processing zone at an equivalent pellet radius is calculated using the formula:

$$
I=\left(\frac{U}{2 \pi k \rho_{1} r}+\frac{U}{\pi \rho_{2} r(\pi+2 k)+2 R_{n}}\right) n \frac{L_{c}}{L_{0}},
$$

where $U$-electrode voltage, $\mathrm{V} ; k$ - the coefficient, taking account of the ratio between the specific electrolyte resistance and the same parameter for pellet material; $p_{2}-$ the specific resistance of the pellet material; $n=f(r, l)-$ the number of pellets along the length $L_{c} ; r$ - the radius of abrasive pellets; $R_{n}$ - the transfer resistance of current flow from the electrolyte to the pellet; $L_{c}$ - the distance between the nozzle section and the processing point; $L_{0}-$ the distance from the nozzle section to the workpiece along the jet axis during the adjustment.

We assume, that the speed of allowance removal $\left(V_{n}\right)$ under the impact of abrasive pellets depends on parameters of an electric field and the distance:

$$
v_{n}=K_{\ni} v_{H} L_{c}^{0,3}
$$

where $K_{\ni}$ is the coefficient, taking account of the time of allowance removal speed growth during stripping the modified layer through anodic workpiece dissolution in the processing zone. For steel parts, it is equal to 1,3-1,5; $v_{H}$ - the speed of allowance removal in the processed workpiece zone, closest to the nozzle; it is calculated in the same way as the law of anodic dissolution for stationary electrodes. 
Taking account of the regularities of allowance removal speed, it is possible to set the time, required to obtain the finished part profile with set parameters of accuracy and surface layer quality.

\section{The system of jet control during multi- axis movement of the machining zone}

The mechanism of allowance removal, discussed above, makes it possible to design the automated system of jet movement, ensuring the required accuracy during finishing of adjoining surfaces of arbitrary shape. The system relies on the mathematical description of the movement of a jet, in which its boundaries are located on the processed section, and the variable material removal is ensured by the change of jet position (as a rule, by the angle of attack), provided the required quality of workpiece surface layer is ensured. In case of small width of the coupling section (edge), the jet axis must be located on the symmetry line of an adjoining profile with coordinates which can also be set by points $\mathrm{X}_{\mathrm{ij}}, \mathrm{Y}_{\mathrm{ij}}, \mathrm{Z}_{\mathrm{ij}}$.

To ensure the coupling of spatial surfaces, the law of allowance removal speed change, depending on the angle of attack, is used as a control parameter. The machining of an arbitrary surface by a jet is possible with real-time control of all movements.

When a jet moves along L-L (fig.2), it is required to control its vector with reference to minimal number of control parameters.

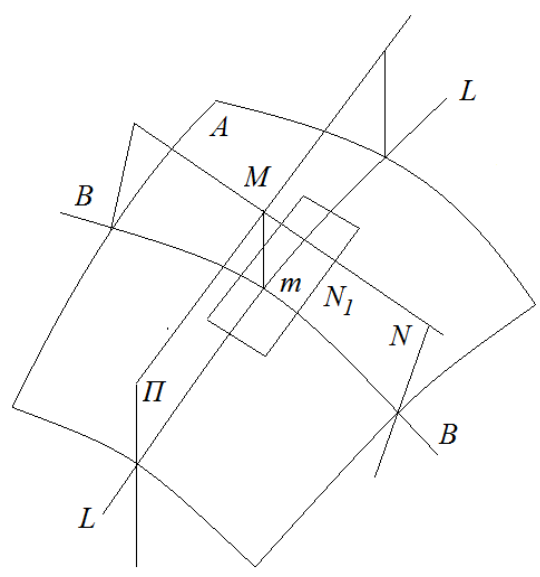

Fig. 2. The scheme of jet movement during geometricallycomplex surface machining:

$m$ - the point of jet contact with a processed zone; $M$ - the nozzle section for the supply of operating medium; $L-L, B-B$ - the generatrices of the processed surface; $N, P, N_{1}$ - the planes in the processing zone

The methodology is developed for calculating the pitch of linear shift and finding the coordinates of a processing point with account of the jet radius $R_{c}$ in the contact point.

$$
\Delta X=\left[1-\frac{R_{c}^{2} x x \cos \alpha}{\left(1+z_{x}^{\prime 2}\right)^{3 / 2}}+\frac{R_{c}^{2} \alpha_{x}^{\prime} z_{x}^{\prime} \sin \alpha}{\left(1+z_{x}^{\prime 2}\right)^{1 / 2}}\right] \Delta x ;
$$

$$
\Delta Y=R_{c}(\theta) \alpha_{x}^{\prime} \cos \alpha \Delta x
$$

$$
\Delta Z=\left[z_{x}^{\prime}+\frac{R_{c} z^{\prime} x z_{x x}^{\prime \prime} \cos \alpha}{\left(1+z_{x}^{\prime 2}\right)^{3 / 2}}+\frac{R_{c} \alpha_{x}^{\prime} \sin \alpha}{\left(1+z_{x}^{\prime 2}\right)^{1 / 2}}\right] \Delta x
$$

where $\dot{\alpha}$ - the slope angle of the processed surface with respect to the jet axis; $x^{\prime}, y^{\prime}, z^{\prime}-$ the change of jet axis coordinates due to variable speed of contact point movement along the line with a curvilinear generatric (the first derivative); $x^{\prime \prime}, y^{\prime \prime}, z^{\prime \prime}$ - the change of jet axis coordinates due to variable acceleration during machining of surface sections of various curvature (the second derivative with respect to the axis specified); $z_{x x}^{\prime \prime}$ indicates the movement along the axis $x$, with variable speed and acceleration.

The machining of the surface, specified by coordinates, is more complicated. These include the engine blades with highly twisted edges.

Provided the angle of tangent slope towards the part profile is constant, then, by analogy with mechanical processing of such surfaces, the position of a point on the processed part zone can be represented as:

$$
X_{i, j}=\frac{x_{i, j} R_{c} \cos \alpha_{i, j}}{\alpha_{i+1, j}-\alpha_{i, j}}\left(\sqrt{1+\alpha^{2}{ }_{i+1, j}}-\sqrt{1+\alpha^{2}{ }_{i, j}}\right),
$$

where $\alpha_{i+1}$ - the angle on the subsequent line.

$$
\begin{gathered}
Y_{i, j}=y_{i} ; \\
Z_{i, j}=\alpha_{i} x_{i, j}+y_{j} \operatorname{tg} \alpha_{i+}+D_{i, j}
\end{gathered}
$$

where $D_{i, j}$ is jet divergence at the nozzle section along the coordinates; $d_{i, j}$ is the divergence in a processing point, shifting along the line with unit vectors.

With account of regularities discovered, the analysis of possible trajectory movements of a jet during combined hydroabrasive finishing has been conducted, using the examples of geometrically-complex surfaces, in which a standard unit is a profile of the airfoil edge and the transfer blade zone of jet and rocket engines, as well as other blade-type machines. The analysis has shown that the reviewed process is most efficient for products with limited tool access, where the jet method has significant advantage.

\section{Conclusion}

The study confirms the possibility of using available and cheap, or current-conducting abrasives in combined finishing, not previously implemented in hydroabrasive machining $[12,13]$. The stable accuracy of $6-8$ quality degrees has been achieved, given the roughness of surface layer for steels is less than $1 \mu \mathrm{m}$, which ensures high- 
quality finishing of parts and transfer zones with profiles of any spatial geometry.

\section{References}

1. The RF Patent № 2465994 MPK B23H5/00. The method of eliminating the impregnation effect during hydroabrasive separation of viscous metals / Patent pending 2011105507: claimed 14.02.2010: published 20.08.2012 The Bulletin № 31.

2. The RF Patent № 2455132 MPK B23H. The method of hydroabrasive separation of viscous metals / Patent pending 2010149206: claimed 30.11.2010: published 10.07.2012. The Bulletin № 19.

3. The RF Patent №2536128 МПК В23H. The method of combine separation of metals / Patent pending 2012116013: claimed 19.04.2012: published. 20.12.2014 . The Bulletin № 35

4. G.V.Barsukov, A.V.Mikheev, The determination of hydroabrasive cutting productivity with account of abrasive grain characteristics, Journal of Engineering. The reference book, № 1, PP.9-14 (2008)

5. B.A. Burnashov, The determination of cutting strength during cutting of material by a highpressure ice-water jet, The Bulletin of Bryansk State Technical University, № 4, PP. 17 - 20 (2008)

6. E.V.Goncharov, V.P.Smolentsev, O.N.Kirillov, The combined separation of metals in the electric field, The fundamental and applied problems of engineering and technology. № 2-5 (292), PP. 10 15 (2012)

7. D.G. Moriss, M. Mazmy, C. Noseda, Creep resistance in a new alloy based on Fe3 $\mathrm{Al}$, Scr. Met et Mater, № 2 - 31, PP. 173 - 178 (1994)

8. V.K. Sikka, S. Viswanathan, C.G. McKaamey, Development and commercialization status of $\mathrm{Fe} 3 \mathrm{Al}$ - based intermetallic alloys, Struct. Intermetallics: Champion, PP. 26 - 30 (1993)

9. V.P.Smolentsev, E.V.Goncharov, The calculation of technological modes of hydroabrasive separation of materials with application of an electrical field, The Bulletin of Voronezh State Technical University, Volume 8, № 4, PP. 130-133 (2012)

10. Y.S.Stepanov, G.V.Barsukov, E.G.Alyushin, The modern technologies of hydro- and hydroabrasive workpiece machining, Science-based technologies in machine-construction, № 6, PP. 11-17 (2012)

11. Y.S.Stepanov, A.P.Cherepenko, M.A.Burnashov, The technology of cutting the technical textile materials and their deriviative compositions, applied in transport machine-construction, The Reference. The Journal of Engineering, № 1, PP. 3 - 6 (1999)

12. Y.S.Stepanov, A.N.Kachanov, MA.Burnashov, The methodology of calculating the technological parameters of cutting non-metal materials by icewater tools, Polzunovsky Vestnik, № 1-2, PP. 276277 (2009)

13. A.G. Suslov, B.M.Bazrov, V.F.Bezyazychnyi et al, Science-based technologies in machine-construction, (Moscow: Mashinostroenie, 2012) 